\title{
Intraperitoneal abscess from perforated diverticulitis with fistualization to extraperitoneal abscess into the scrotum: a case report
}

\author{
Julia T. Scali ${ }^{1}$, Young G. Son ${ }^{2 *}$, lan T. Madison³ , Benjamin A. Fink ${ }^{1}$ and Thomas J. Mueller ${ }^{4}$
}

\begin{abstract}
Background: Scrotal abscesses are common skin abscesses seen in the general population; however, intraperitoneal abscesses tracking into the scrotum are rare. Intraperitoneal and retroperitoneal abscesses contiguous with the scrotum have been reported in the literature in specific populations. Wound cultures can aid in differentiating the source of the infections. Recurrent abscesses have been observed in high-risk populations, such as those with malignancy or who are immunocompromised.
\end{abstract}

Case presentation: We present a 71-year-old male with pericolonic abscess following perforated diverticulitis. The abscess was drained with interventional radiology and was complicated by an extraperitoneal abscess that tracked to the scrotum. Incision and drainage of the extraperitoneal abscess and the scrotal abscess were required with intravenous antibiotic therapy. The abscess tracking into the scrotum is reported without evidence of patent processus vaginalis.

Conclusion: We conclude that an intraperitoneal abscess can track to the scrotum through extraperitoneal fascial planes in the absence of a patent processus vaginalis. We show that extraperitoneal abscess spread to the scrotum is possible, with wound cultures helping to differentiate the source of the infection. High-risk patients with recurrent abscesses can also be susceptible to contiguous spread.

Keywords: Scrotal abscess, Diverticulitis, Diverticular abscess, Intraperitoneal abscess, Extraperitoneal abscess, Processus vaginalis, Pericolonic abscess, Case report

\section{Background}

Scrotal abscesses are common and associated with trauma, urinary tract infection, diabetes, immunocompromised state, epididymo-orchitis, and poor hygiene [1]. Scrotal abscesses draining from an intraperitoneal source are rare and usually not seen in adults. In children, reports of intraperitoneal abscesses draining to the

\footnotetext{
*Correspondence: Youngso@pcom.edu

2 Jefferson New Jersey Urology Resident PGY 2, 1 Medical Drive Suite 162,

Stratford, NJ 08084, United States

Full list of author information is available at the end of the article
}

scrotum have been described via a patent processus vaginalis following complicated appendicitis [2].

The testes are formed in the abdomen and descend through the peritoneum, abdominal wall, and the inguinal canal via the processus vaginalis to reach the scrotum [3]. The inguinal canal contains the processus vaginalis derived from the peritoneum, which eventually obliterates to confine the testes. Closure prevents communication between the scrotum and intraperitoneal space. Failure of closure, called patent processus vaginalis, has been a risk factor for hydroceles, inguinal hernias, and scrotal abscesses in children [3, 4]. An adult patient 
with perforated diverticulitis complicated by pericolonic abscess with extension into the scrotum is rare, especially without evidence of a patent processus vaginalis.

\section{Case presentation}

We present a 71-year-old-male with a past medical history of hypertension, diabetes mellitus, and gout who presented to the emergency department for evaluation of worsening left lower quadrant abdominal pain that began two days prior to presentation. On arrival, the patient had a creatinine of $2.56 \mathrm{mg}$ (milligram)/dL (deciliter), white blood cell count of $29.7 \times 10^{3}$ per uL (microliter), and lactate of $2.7 \mathrm{mg} / \mathrm{dL}$. The patient was started on piperacillin-tazobactam in the emergency department for a potential intraperitoneal infection. Computed tomography $(\mathrm{CT})$ with contrast was recommended but was unable to be obtained due to patient allergy to intravenous contrast and acute kidney injury. CT abdomen and pelvis (CTAP) without contrast revealed complicated descending colonic diverticulitis associated with adjacent linear air and fluid-filled collection, extending from the medial aspect of the lower descending colon into the anterior aspect of the peritoneal cavity and greater omentum against the adjacent rectus abdominis muscle (Fig. 1).

Interventional radiology (IR) performed a CT-guided drainage of the abdominal abscess with placement of an 8-French percutaneous drain into the abdominal collection. On postoperative day one, the patient began to complain of left testicular pain and scrotal swelling. Scrotal ultrasound revealed findings suspicious for a developing, ill-defined, left-sided scrotal wall complex abscess (Fig. 2). A repeat CTAP down to mid-thigh without contrast showed a scrotal decubitus and soft tissue defect, concerning for scrotal abscess development, but with improvement in the previously drained anterior abdominal fluid collection. Bedside scrotal incision and drainage were performed and cultures from the collection grew coagulase-negative Staphylococcus with mixed gram negative and positive flora. Three days after bedside incision and drainage, the patient was switched from piperacillin-tazobactam to meropenem due to an increase in $\mathrm{WBC}$ count from $20.4 \times 10^{3}$ per $\mathrm{uL}$ to $31.5 \times 10^{3}$ per uL.

One week after abdominal abscess drainage, the patient's white blood cell count persisted, with a maximum of $35.7 \times 10^{3}$ per uL. After starting meropenem, the patient's white blood cell count began to decrease, and with the infection apparently resolving, the infectious disease team transitioned the patient to oral amoxicillin-clavulanate.

Eleven days following the IR procedure, excessive purulent discharge was noted from the scrotal incision. Urology was consulted again and noted insufficient scrotal

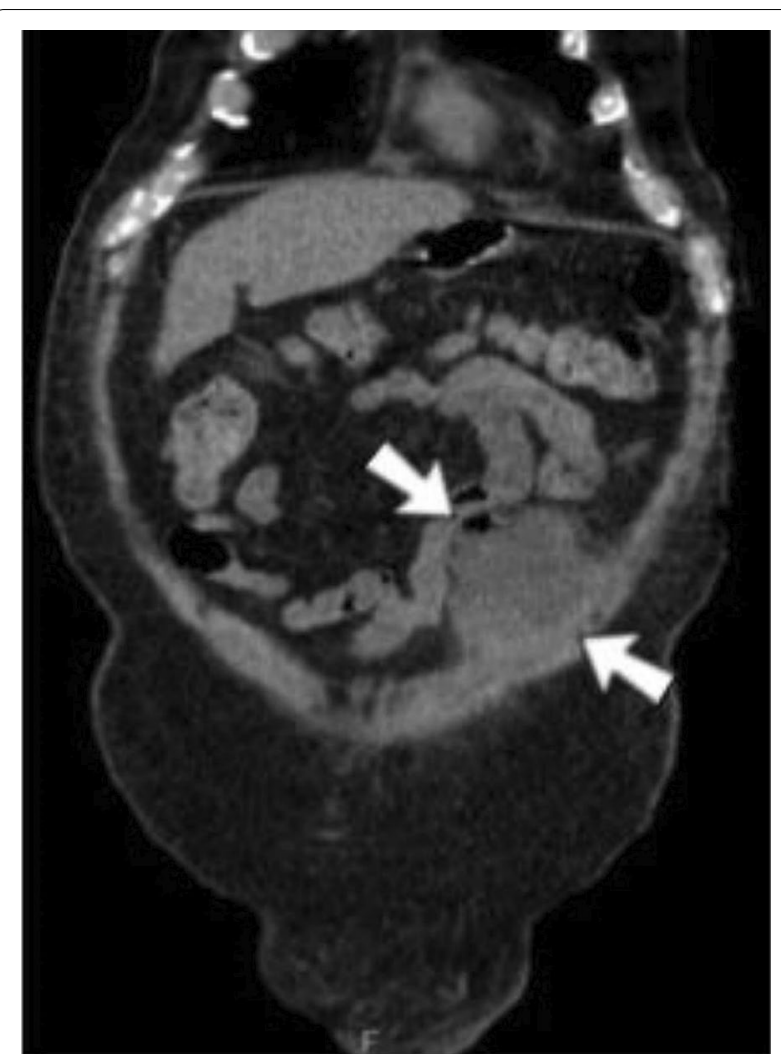

Fig. 1 Coronal plane CTAP without contrast showing complicated descending colonic diverticulitis associated with adjacent developing localized air leak and abscess formation (arrows)

packing, which was presumed to be the reason for the persistent abscess. Bedside drainage was performed again, and repeat cultures showed various enteric organisms, including multidrug-resistant Enterobacter cloacae, Eikenella species, and yeast. In addition, a drain study with fluoroscopy demonstrated the presence of a fistulous tract to the colon (Fig. 3). Repeat CTAP showed that the previously drained intraperitoneal abscess had resolved; however, there was an interval development of a large anterior abdominal wall abscess measuring $26 \times 4 \mathrm{~cm}(\mathrm{~cm})$ that extended into the scrotum (Fig. 4). CTAP also showed persistent thickening at the junction of sigmoid and descending colon that was highly suspicious for a neoplastic mass.

The patient required surgical incision and drainage and was taken to the operating room for abscess drainage with the general surgery team. A suprapubic transverse incision and a left lower quadrant incision were made, and superficial subcutaneous tissue was dissected until the abscess cavity was entered. The peritoneal cavity was revealed to communicate with the anterior abdominal wall abscess, which was continuous with the scrotal abscess cavity. The following day, the patient was taken 

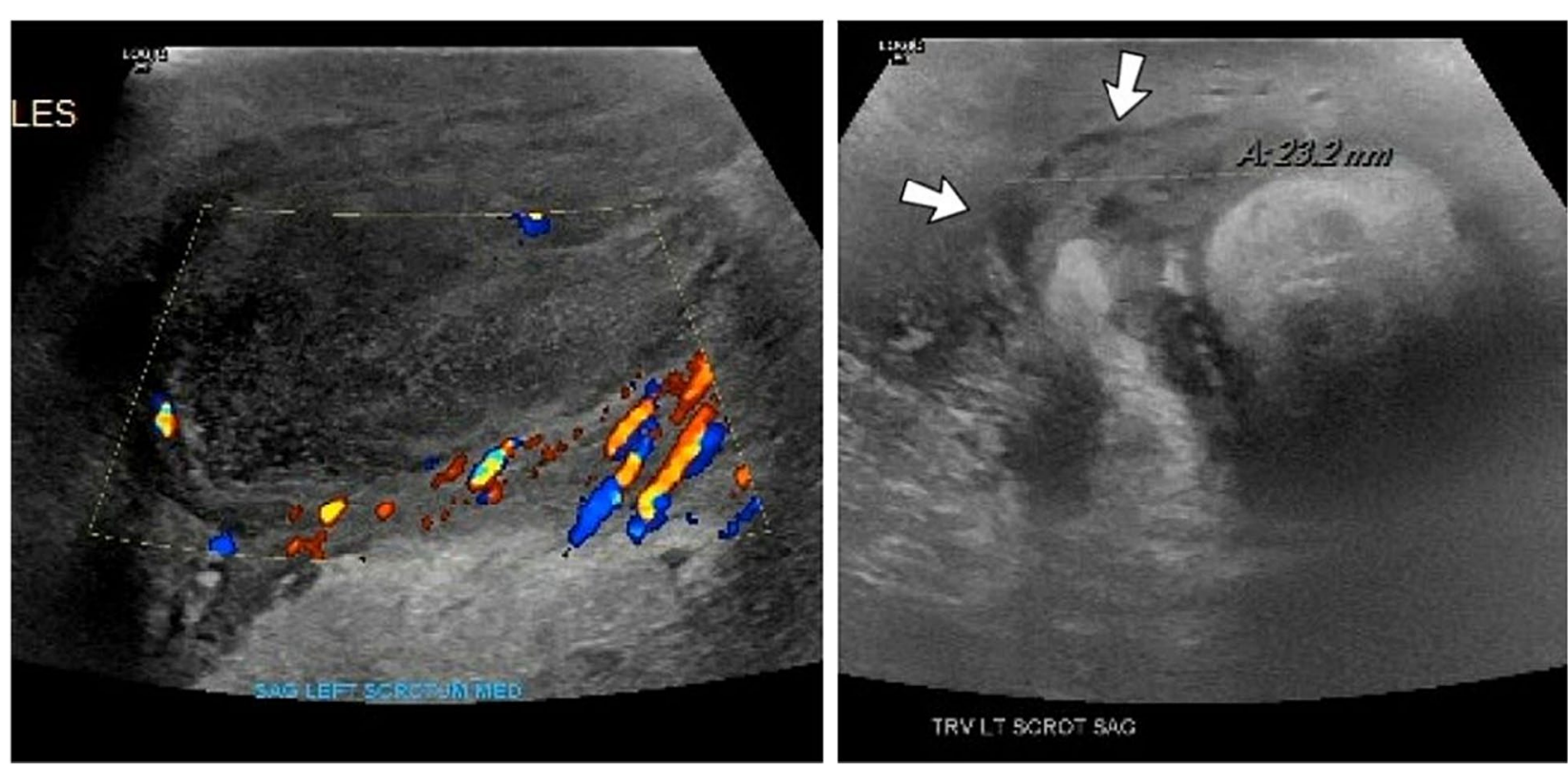

Fig. 2 Sagittal view (left) and transverse view (right) of left scrotal ultrasound showing mild to moderate degree of bilateral, left-sided scrotal wall edematous changes with increased color Doppler flow (left) associated with an ill-defined fluid collection (right with arrows) developing within the left-sided scrotal wall outside the tunica vaginalis. Transverse diameter measured approximately $2.3 \mathrm{~cm}$

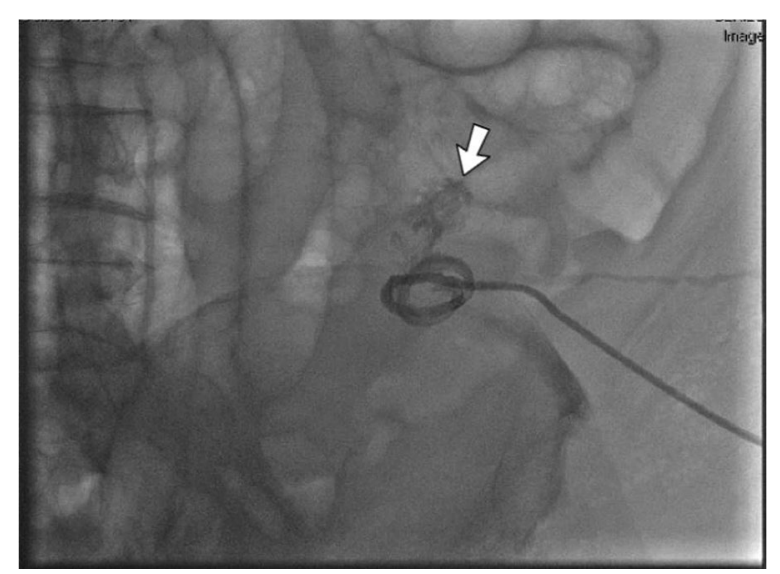

Fig. 3 IR drain study with fluoroscopy demonstrating a fistulous tract to the colon (arrow), with absent intraperitoneal fluid collection

back to the operating room to have the two abdominal incisions connected for negative pressure wound therapy and minimal debridement of residual necrotic tissue was performed (Fig. 5). The patient was brought to the operating room every two days for a total of three negative pressure wound dressing changes and debridement. On the final operating room day, separate negative pressure wound sponges for the abdominal cavity and the left scrotum were used. The patient was eventually transitioned to wet-to-dry dressing changes in the scrotum and continuation of the negative pressure wound dressing changes in the abdominal cavity.

The patient underwent a repeat drain study for the 8 French percutaneous drain that showed resolution of the intraabdominal abscess without collection or fistula. The percutaneous drain was removed, and the antibiotic regimen was de-escalated to oral ciprofloxacin and metronidazole. Creatinine returned to baseline, white blood cell counts normalized $\left(9.7 \times 10^{3}\right.$ per $\mathrm{uL}$ on discharge), and infectious disease recommended oral antibiotic regimen for discharge to acute rehab.

\section{Conclusions}

There were previously reported cases of retroperitoneal and intraperitoneal abscesses that tracked into the scrotum. Factors such as chronic inflammation with abscess formation can track through fascial planes to the most dependent portion of the body. We hypothesize that there can be another track that can be a direct extension from the extraperitoneal space to the scrotum through the various fascial planes.

\section{Discussion}

The initial abscess started pericolonically following perforated diverticulitis in the left anterior peritoneal space abutting the rectus abdominis. Fistulas usually develop secondary to a foreign body, radiation, inflammation, epithelialization, neoplasm, or distal 


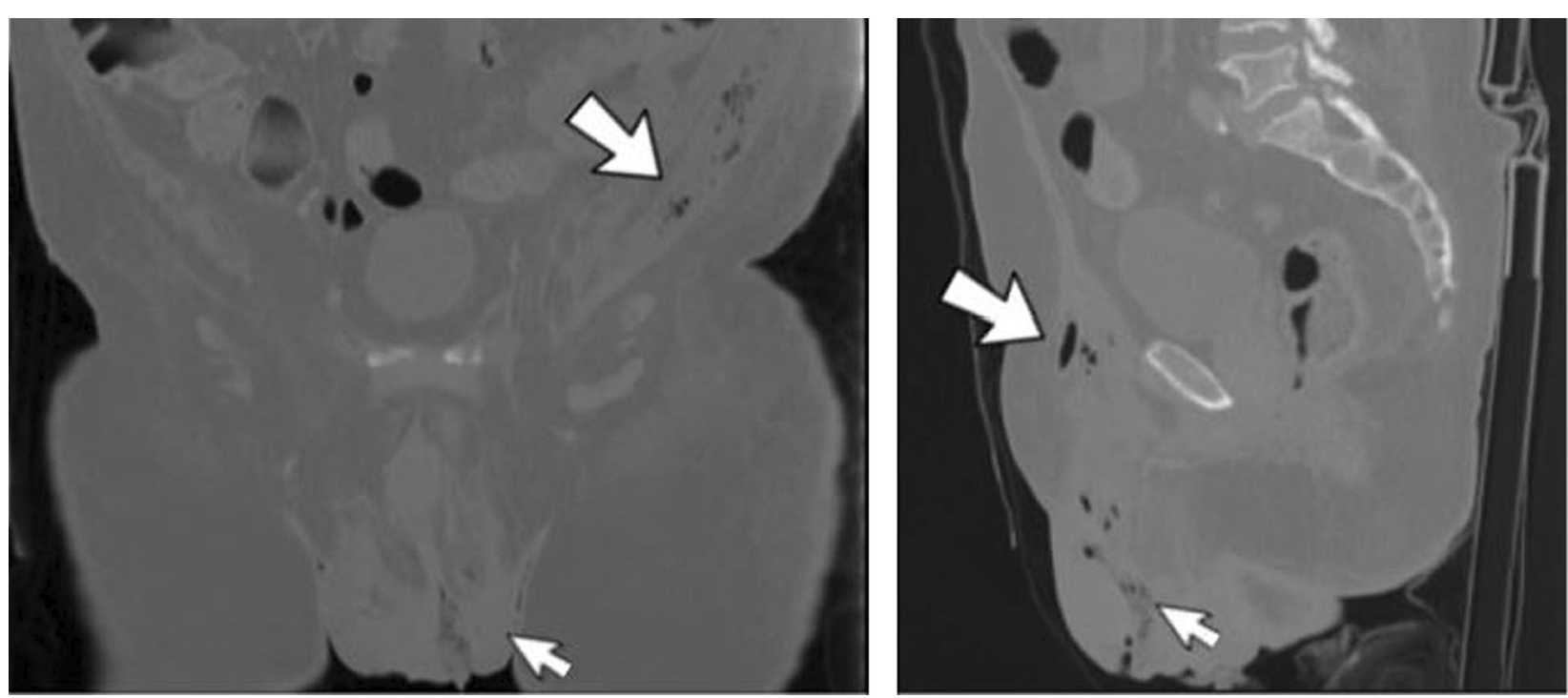

Fig. 4 Coronal plane (left) and sagittal plane (right) CTAP without contrast showing development of a large anterior abdominal wall abscess measuring $26-\mathrm{cm} \times 4-\mathrm{cm}$ (thick arrows) extending into the scrotum (thin arrows)
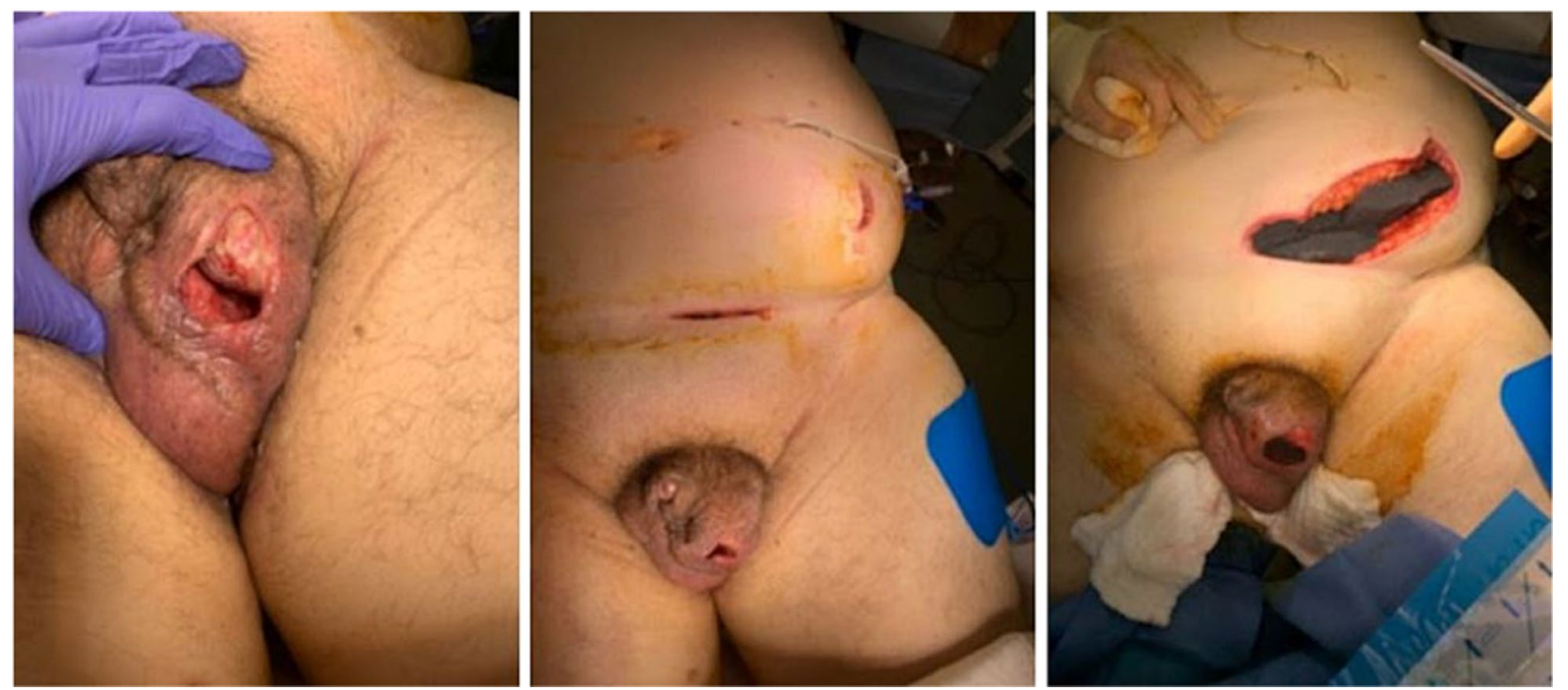

Fig. 5 Intraoperative images of scrotal incision (left), suprapubic and left lower quadrant abdominal incisions (middle) that were subsequently connected (right) for negative pressure wound therapy

obstruction [5]. Colonic inflammation from appendicitis and diverticular disease has also been associated with fistula formation [6]. Our patient had several of these risk factors, including inflammation from diverticulitis, the introduction of a foreign body by IR, and colonic abscess formation. We hypothesize that the intraperitoneal sigmoid colon diverticular abscess was the root cause, and the secondary abscess that was in the anterior extraperitoneal space adjacent to the rectus abdominis was the abscess that drained into the left scrotum.

Abscess formation is a well-known complication of perforated diverticulitis. It often occurs intraperitoneally and can typically be treated with antibiotics alone if the abscess is less than three centimeters. Percutaneous abscess drainage (PAD) is required for larger abscesses [7]. One study that systematically reviewed the outcomes after treatment of acute diverticulitis with 
abscess formation showed that of patients treated nonoperatively (antibiotics or PAD), 25\% experienced a recurrent episode during long-term follow-up, and of the patients undergoing PAD, 2.5\% experienced procedurerelated complications [8].

The most common organisms that cause cutaneous abscesses are gram-positive bacteria, such as Staphylococcus aureus, which are found on flora of human skin and mucous membranes [9]. However, anaerobic bacteria are frequently isolated, with the true prevalence of these infections undetermined due to slow growth, difficulty in culturing, and polymicrobial nature of the infection [10]. Abscesses associated with diverticulitis are due to diverticular microperforations and extravasation of enteric commensals, with Escherichia coli and Bacteroides species being the most frequently isolated [11].

The evolution of cultures from the scrotal abscess site supports the hypothesis that enteric spread caused the secondary abscess. Cultures of the scrotal abscess from the initial bedside incision and drainage showed a predominant growth of coagulase-negative staphylococcus, gram-positive cocci, and mixed anaerobic bacteria. The presence of coagulase-negative staphylococcus indicates that there was likely floral contamination that contributed to this initial abscess formation [12]. Subsequent cultures showed growth of Enterobacter cloacae, which has been the most common cause of nosocomial infection within the last decade [13]. This shift in culture suggests that the initial scrotal abscess was unrelated to the pericolonic abscess; however, the secondary scrotal abscess was associated with the intraperitoneal abscess as the isolated species were related to organisms that typically reside within the gastrointestinal tract. We can postulate that the formation of a fistulous tract between the peritoneum and abdominal wall allowed for direct extension, which was confirmed with $\mathrm{CT}$ imaging and intraoperatively.

The fistulous tract that developed anteriorly dissected through the transversalis fascia, rectus sheath, and invested in Scarpa's fascia before spreading inferiorly into the scrotum. Scarpa's fascia is contiguous with the Dartos fascia below the pubic symphysis which can provide a direct route from the anterior abdominal wall into the scrotum for potential spread [14]. A retroperitoneal tract has also been hypothesized to be a cause of abscess tracking to the scrotum. A retroperitoneal abscess formation tracking to the scrotum has been described in the literature. An ascending and descending colonic perforation with subsequent spread along the retroperitoneal fascial planes resulting in scrotal abscess with fecal contents in the scrotum has been reported [15]. The postulated mechanism is that the inflammation from the retroperitoneum caused the abscess to dissect through the transversalis fascia through the deep inguinal ring. Another pathway to the scrotum is with a patent processus vaginalis.

Documented scrotal abscess formation from an intraperitoneal source most commonly occurs in the pediatric population, often associated with a patent processus vaginalis. The patent processus vaginalis serves as a communication between the peritoneal cavity and the scrotum, with several case reports describing a phenomenon of peritoneal extension of an abscess associated with perforated appendicitis. The presenting symptom of appendicitis in these cases is scrotal swelling in young boys [4]. A patent processus vaginalis has been estimated to be present in $80-95 \%$ of newborn males, with $15-37 \%$ of those persisting into adulthood [2-4]. However, a patent processus vaginalis will typically close within the first 24 months of life and is unlikely to cause a scrotal abscess via intraperitoneal extension in an adult. Instead, scrotal extension of an intraperitoneal abscess in an adult can be caused by direct extension [16]. It is rare for fluid collection to drain into the scrotum without a patent processus vaginalis, especially in adults. We conclude that there are extraperitoneal planes that can lead to scrotal abscess collection from an abdominal source.

\section{Patient perspective}

The patient was hospitalized for a total of 27 days with two bedside scrotal incision and drainages, three interventional radiology procedures, and four procedures in the operating room. The patient tolerated the procedures well and was discharged to acute rehab on day 28 . On follow-up, the patient was gracious for the care he received during his hospital stay and continued negative pressure wound therapy at home.

\section{Abbreviations}

mg: Milligrams; dL: Deciliter; uL: Microliter; CT: Computed tomography; CTAP: Computed tomography of abdomen and pelvis; IR: Interventional radiology; $\mathrm{cm}$ : Centimeter; PAD: Percutaneous abscess drainage.

\section{Acknowledgements \\ None.}

\section{Authors' contributions}

JTS and YGS analyzed the patient data and wrote the preliminary draft. ITM, BAF, TJM provided expertise and edited the manuscript. All authors read and approved the final manuscript.

\section{Funding}

No funding to declare for this article.

Availability of data and materials

Data sharing is not applicable to this article as no datasets were generated or analyzed during the current study. 


\section{Declarations}

Ethics approval and consent to participate

Written informed consent to participate was obtained from the patient of this case report. A copy of the written consent is available for review by the Editor of this journal.

\section{Consent for publication}

Written informed consent was obtained from the patient for publication of this case report and any accompanying images. A copy of the written consent is available for review by the Editor of this journal.

\section{Competing interests}

The authors declare that they have no competing interests.

\section{Author details}

'Rowan University School of Osteopathic Medicine, 42 E Laurel Rd, Stratford, NJ 08084, United States. ${ }^{2}$ Jefferson New Jersey Urology Resident PGY 2, 1 Medical Drive Suite 162, Stratford, NJ 08084, United States. ${ }^{3}$ Philadelphia College of Osteopathic Medicine, 4170 City Ave, Philadelphia, PA 19131, United States. ${ }^{4}$ Jefferson New Jersey Urology Residency Program Director, 1 Medical Drive Suite 162, Stratford, NJ 08084, United States.

Received: 4 October 2021 Accepted: 25 October 2021

Published online: 04 November 2021

\section{References}

1. Kaye KS, Petty LA, Shorr AF, Zilberberg MD (2019) Current epidemiology, etiology, and burden of acute skin infections in the United States. Clin Infect Dis 68:193-199. https://doi.org/10.1093/cid/ciz002

2. Keyes J, Casas-Melley AT, Liu C, Epelman MS, Ellsworth PI (2020) Scrotal abscess after a perforated appendicitis. J Surg Case Rep. https://doi.org/ 10.1093/jscr/rjaa058

3. Tiwana MS, Leslie SW (2021) Anatomy, abdomen and pelvis, testicle. In: StatPearls Internet, Treasure Island (FL): StatPearls Publishing 202129261881

4. Rahman N, Lakhoo K (2009) Patent processus vaginalis: a window to the abdomen. Afr J Paediatr Surg 6:116-117
5. Cowan KB, Cassaro S (2021) Enterocutaneous Fistula. In: StatPearls [Internet]. Treasure Island (FL): StatPearls Publishing. PMID: 29083609 https:// www.ncbi.nlm.nih.gov/books/NBK459129/

6. Schecter P, Hirshberg A, Chang S et al (2009) Enteric fistulas: principles of management. J Am Coll Surg 209:484-491. https://doi.org/10.1016/j. jamcollsurg.2009.05.025

7. Mali J, Mentula P, Leppäniemi A et al (2019) Determinants of treatment and outcomes of diverticular abscesses. World J Emerg Surg 14:31

8. Gregersen R, Mortensen LQ, Burcharth J, Pommergaard HC, Rosenberg J (2016) Treatment of patients with acute colonic diverticulitis complicated by abscess formation: a systematic review. Int J Surg 35:201-208. https:// doi.org/10.1016/j.ijsu.2016.10.006

9. Malih R, Alrubea F, Hindi N et al (2020) Bacterial skin abscess. Staphylococcus Aureus. https://doi.org/10.5772/intechopen.91657

10. Brook I (2004) Urinary tract and genito-urinary suppurative infections due to anaerobic bacteria. Int J Urol 11:133-141

11. Chow AW (1994) Appendicitis and diverticulitis. In: Hoeprich PD, Jordan MC, Ronald AR (eds) Infectious diseases: a treatise of infectious processes. JB Lippincott, Philadelphia, pp 878-881

12. Hall KK, Lyman JA (2006) Updated review of blood culture contamination. Clin Microbiol Rev 19:788-802. https://doi.org/10.1128/CMR.00062-05

13. Davin-Regli A, Pagès JM (2015) Enterobacter aerogenes and Enterobacter cloacae; versatile bacterial pathogens confronting antibiotic treatment. Front Microbiol. https://doi.org/10.3389/fmicb.2015.00392

14. Joshi R, Duong H (2021) Anatomy, Abdomen and Pelvis, Scarpa Fascia. In: StatPearls [Internet, Treasure Island (FL): StatPearls Publishing. 202131985930

15. Bahadur A, Singh N, Kashmira M et al (2021) Fecal scrotal abscess secondary to spontaneous retroperitoneal perforation of ascending colon. Case Rep Med. https://doi.org/10.1155/2021/6658083

16. Rothenbuehler JM, Oertli D, Harder F (1993) Extraperitoneal manifestation of perforated diverticulitis. Dig Dis Sci 38:1985-1988

\section{Publisher's Note}

Springer Nature remains neutral with regard to jurisdictional claims in published maps and institutional affiliations.

\section{Submit your manuscript to a SpringerOpen ${ }^{\circ}$ journal and benefit from:}

- Convenient online submission

- Rigorous peer review

- Open access: articles freely available online

- High visibility within the field

Retaining the copyright to your article

Submit your next manuscript at $\boldsymbol{\nabla}$ springeropen.com 